\title{
Predicting the Occurrence of Variants in RAG1 and RAG2
}

\section{Dylan Lawless $^{1}$ (D) • Hana Lango Allen ${ }^{3,4}$ - James Thaventhiran ${ }^{5}$. NIHR BioResource-Rare Diseases Consortium ${ }^{3}$.

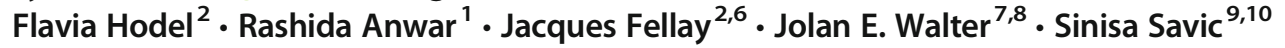

Received: 25 September 2018 / Accepted: 15 July 2019 / Published online: 6 August 2019

(C) The Author(s) 2019

\begin{abstract}
While widespread genome sequencing ushers in a new era of preventive medicine, the tools for predictive genomics are still lacking. Time and resource limitations mean that human diseases remain uncharacterized because of an inability to predict clinically relevant genetic variants. A strategy of targeting highly conserved protein regions is used commonly in functional studies. However, this benefit is lost for rare diseases where the attributable genes are mostly conserved. An immunological disorder exemplifying this challenge occurs through damaging mutations in $R A G 1$ and $R A G 2$ which presents at an early age with a distinct phenotype of life-threatening immunodeficiency or autoimmunity. Many tools exist for variant pathogenicity prediction, but these cannot account for the probability of variant occurrence. Here, we present a method that predicts the likelihood of mutation for every amino acid residue in the RAG1 and RAG2 proteins. Population genetics data from approximately 146,000 individuals was used for rare variant analysis. Forty-four known pathogenic variants reported in patients and recombination activity measurements from 110 RAG1/2 mutants were used to validate calculated scores. Probabilities were compared with 98 currently known human cases of disease. A genome sequence dataset of 558 patients who have primary immunodeficiency but that are negative for RAG deficiency were also used as validation controls. We compared the difference between mutation likelihood and pathogenicity prediction. Our method builds a map of most probable mutations allowing pre-emptive functional analysis. This method may be applied to other diseases with hopes of improving preparedness for clinical diagnosis.
\end{abstract}

Keywords Recombination activating genes 1 and $2($ RAG1, RAG2) $\cdot$ pathogenic variant $\cdot$ genomics $\cdot$ predictive

\section{Abbreviations \\ BCR B cell receptor \\ CADD Combined annotation dependent depletion}

Electronic supplementary material The online version of this article (https://doi.org/10.1007/s10875-019-00670-z) contains supplementary material, which is available to authorized users.

Dylan Lawless

Dylan.Lawless@epfl.ch

Sinisa Savic

S.Savic@leeds.ac.uk

1 Leeds Institute of Biomedical and Clinical Sciences, St James's University Hospital, University of Leeds, Wellcome Trust Brenner Building, Beckett Street, Leeds, UK

2 Global Health Institute, School of Life Sciences, École Polytechnique Fédérale de Lausanne, 1015 Lausanne, Switzerland

3 NIHR BioResource, Cambridge University Hospitals, Cambridge Biomedical Campus, Cambridge CB20QQ, UK

4 Department of Haematology, University of Cambridge, Cambridge Biomedical Campus, Cambridge CB20XY, UK
CID-G/A Combined immunodeficiency with granuloma and/or autoimmunity

GWAS Genome-wide association studies
5 MRC Toxicology Unit, School of Biological Sciences, University of Cambridge, Cambridge, UK

6 Swiss Institute of Bioinformatics, 1015 Lausanne, Switzerland

7 University of South Florida and Johns Hopkins All Children's Hospital, Saint Petersburg, FL, USA

8 Division of Allergy Immunology, Massachusetts General Hospital for Children, Boston, MA, USA

9 Department of Clinical Immunology and Allergy, St James's University Hospital, Beckett Street, Leeds, UK

10 National Institute for Health Research Leeds Musculoskeletal Biomedical Research Centre and Leeds Institute of Rheumatic and Musculoskeletal Medicine, St James's University Hospital, Wellcome Trust Brenner Building, Beckett Street, Leeds, UK 


$\begin{array}{ll}\text { HGMD } & \text { Human gene mutation database } \\ \text { Mr. } & \text { Mutation rate } \\ \text { MRF } & \text { Mutation rate residue frequency } \\ \text { PID } & \text { Primary immunodeficiency } \\ \text { pLI } & \text { Probability of being loss-of-function intolerant } \\ \text { RAG1 } & \text { Recombination activating gene 1 } \\ \text { Rf } & \text { Residue frequency } \\ \text { RNH } & \text { RNase H } \\ \text { RSS } & \text { Recombination signal sequence } \\ \text { SCID } & \text { Severe combined immunodeficiency } \\ \text { TCR } & \text { T cell receptor }\end{array}$

\section{Introduction}

Costs associated with genomic investigations continue to reduce [1], while the richness of data generated increases. Globally, the adoption of wide-scale genome sequencing implies that all newborn infants may receive screening for pathogenic genetic variants in an asymptomatic stage, preemptively [2]. The one dimensionality of individual genomes is now being expanded by the possibility of massive parallel sequencing for somatic variant analysis and by single-cell or lineage-specific genotyping, culminating in a genotype spectrum. In whole blood, virtually every nucleotide position may be mutated across $10^{5}$ cells [3]. Mapping one's genotype across multiple cell types and at several periods during a person's life may soon be feasible [4]. Such genotype snapshots might allow for prediction and tracking of somatic, epigenetic, and transcriptomic profiling.

The predictive value of genomic screening highly depends on the computation tools used for data analysis and its correlation with functional assays or prior clinical experience. Interpretation of that data is especially challenging for rare human genetic disorders; candidate disease-causing variants that are predicted as pathogenic often require complex functional investigations to confirm their significance. There is a need for predictive genomic modelling with aims to provide reliable guidance for therapeutic intervention for patients harboring genetic defects for life-threatening disease before the illness becomes clinically significant.

The study of predictive genomics is exemplified by consideration of gene essentiality, accomplished by observing intolerance to loss-of-function variants. Several gene essentiality scoring methods are available for both the coding and non-coding genome [5]. Approximately 3000 human genes cannot tolerate the loss of one allele [5]. The greatest hurdle in monogenic disease is the interpretation of variants of unknown significance while functional validation is a major time and cost investment for laboratories investigating rare disease.

Severe, life-threatening immune diseases are caused by genetic variations in almost 300 genes [6,7]; however, only a small percentage of disease-causing variants have been characterized using functional studies. Several robust tools are in common usage for predicting variant pathogenicity. Compared with methods for pathogenicity prediction, a void remains for predicting mutation probability, essential for efficient pre-emptive validation. Our investigation aims to apply predictive genomics as a tool to identify genetic variants that are most likely to be seen in patient cohorts.

We present the first application of our novel approach of predictive genomics using Recombination activating gene 1 (RAG1) and RAG2 deficiency as a model for a rare primary immunodeficiency (PID) caused by autosomal recessive variants. RAG1 and RAG2 encode lymphoid-specific proteins that are essential for $\mathrm{V}(\mathrm{D}) \mathrm{J}$ recombination. This genetic recombination mechanism is essential for a robust immune response by diversification of the $\mathrm{T}$ and $\mathrm{B}$ cell repertoire in the thymus and bone marrow, respectively $[8,9]$. Deficiency of RAG1 [10] and RAG2 [11] in mice causes inhibition of B and T cell development. Schwarz et al. [12] formed the first publication reporting that RAG mutations in humans cause severe combined immunodeficiency (SCID), and deficiency in peripheral $\mathrm{B}$ and $\mathrm{T}$ cells. Patient studies identified a form of immune dysregulation known as Omenn syndrome [13, 14]. The patient phenotype includes multi-organ infiltration with oligoclonal, activated $\mathrm{T}$ cells. The first reported cases of Omenn syndrome identified infants with hypomorphic RAG variants which retained partial recombination activity [15]. RAG deficiency can be measured by in vitro quantification of recombination activity [16-18]. Hypomorphic RAG1 and $R A G 2$ mutations, responsible for residual V(D)J recombination activity (on average 5-30\%), result in a distinct phenotype of combined immunodeficiency with granuloma and/or autoimmunity (CID-G/A) [2, 19, 20].

Human RAG deficiency has traditionally been identified at very early ages due to the rapid drop of maternally acquired antibody in the first six months of life. A loss of adequate lymphocyte development quickly results in compromised immune responses. More recently, we have found that RAG deficiency is also found for some adults living with PID [16].

$R A G 1$ and $R A G 2$ are highly conserved genes, but disease is only reported with autosomal recessive inheritance. Only $44 \%$ of amino acids in RAG1 and RAG2 are reported as mutated on GnomAD, and functional validation of candidate variants is difficult [21]. Pre-emptive selection of residues for functional validation is a major challenge; a selection based on low allele frequency alone is infeasible since the majority of each gene is highly conserved. A shortened time between genetic analysis and diagnosis means that treatments may be delivered earlier. RAG deficiency may present with diverse phenotypes, and treatment strategies vary. With such tools, early intervention may be prompted. Some patients could benefit from hematopoietic stem cell transplant 
[22] when necessary, while others may be provided mechanism-based treatment [23]. Here, we provide a new method for predictive scoring that was validated against groups of functional assay values, human disease cases, and population genetics data. We present the list of variants most likely seen as future determinants of RAG deficiency, meriting functional investigation.

\section{Methods}

\section{Population Genetics and Data Sources}

GnomAD (version r2.0.2) [21] was queried for the canonical transcripts of RAG1 and RAG2 from population genetics data of approximately 146,000 individuals; ENST00000299440 (RAG1) 1586 variants, GRCh37 11:36532259-36614706 and ENST00000311485 (RAG2) 831 variants, GRCh37 11:36597124 - 36619829. Data was filtered to contain the variant effect identifiers: frameshift, inframe deletion, inframe insertion, missense, stop lost, or stop gained. Reference transcripts were sourced from Ensembl in the FASTA format amino acid sequence for transcript RAG1201 ENST00000299440.5 [HGNC:9831] and transcript RAG2-201 ENST00000311485.7 [HGNC:9832]. These sequences were converted to their three-letter code format using One to Three from the Sequence Manipulation Suite (SMS2) [24]. Combined Annotation Dependent Depletion (CADD) scores were sourced from https://cadd.gs. washington.edu/download (Nov 2018) and are reported by Kircher et al. [25]. The dataset used was "All possible SNVs" from whole-genome data, from which we extracted the data for coding regions of RAG1 and RAG2. We used the Human Gene Mutation Database (HGMD) from the Institute of Medical Genetics in Cardiff as a pre-defined source of known RAG deficiency cases http://www.hgmd.cf.ac.uk/ ac/index.php [26] (Feb 2019, free access version to NM 000448.2). Data was formatted into CSV and imported into $\mathrm{R}$ for combined analysis with PHRED-scaled CADD scores and the main data frame. The crystal structure render of DNA-bound RAG complex was produced with data from RCSB Protein Data Bank (3jbw.pdb) [27]. Structures were visualized using the software VMD from the Theoretical and Computational Biophysics Group [28], imaged with Tachyon rendering [29], and color mapped using our scoring method.

\section{Data Processing}

The population genetics input dataset used GnomAD variant allele frequencies and reference sequences processed as CSV files and cleaned and sorted to contain only amino acid codes, residue numbers, alternate residues, alternate allele frequencies, and a score of 0 or 1 to indicate presence or absence of variants where 1 represented none reported. An annotation column was also provided to label where multiple alternate variants existed. Statistics and calculation steps are listed in order in Supplemental Tables E3-E8.

The percentage of conserved residues was calculated (55.99\% of amino acids contained no reported variants in RAG1, 55.98\% in RAG2 (Table E4)). Basic protein statistics were generated using canonical reference transcript sequences of RAG1 and RAG2 with the SMS2 tool Protein Stats [24]. The resulting pattern percentage value was converted to a frequency (decimal $0-1$ ) based on the number of residues per protein to generate the residue frequency $(R f)$. The $R f$ values were found for both proteins as shown in Table E5 and summarized in Table E6.

The count of variants per residue was found for both proteins, and the mutation rates $(\mathrm{Mr})$ per residue were calculated as shown in Table E7. $M r$ was found by counting the number of mutations per residue in a window, sized to contain each protein individually. For genome-wide application, the window size may be increased or decreased. In this case, the window consisted of only the coding regions. The $M r$ values were then converted to frequencies based on the number of residues per protein. Separate, and overlapping, windows could also be used based on genome phase data and regions of linkage disequilibrium to account for non-random association of alleles at different loci; this might be particularly important for disorders with multiple genetic determinants.

The $M r$ and $R f$ multiply to give the raw mutation rate residue frequency (MRF) value (Table E8). This value is also shown in Tables 1 and E1. Our investigation used a Boolean score $C$ to account for the presence or absence of a mutation in the general population, 0 for any variant existing in the population and 1 for conserved residues. $C \times M r \times R f$, in our case, produced the MRF score for conserved residues. Figure 1a illustrates the raw MRF as a histogram and the MRF, after applying $\mathrm{C}$, as a heatmap.

An important consideration for future application is whether to use this Boolean score or instead use a discrete variable which accounts for the true allele frequency in the general population. In the clinical setting, the likelihood of de novo mutations and inherited mutations have different impacts when considering recessive and dominant diseases. A patient is more likely to inherit a variant that exists even at a very low frequency than to acquire a random de novo mutation. Therefore, a value representing an allele frequency may be used to replace $C$ in many investigations, particularly when considering variants that exist at low rates. PRHEDscaled CADD score data consisted of nucleotide level values. For comparison with MRF, the median CADD scores were averaged per codon as demonstrated in Supplemental text. A summary of data processing and analysis is illustrated in Fig. E1. 
Table 1 MRF likelihood scores for variants functionally assayed to date [16-18]. Increased MRF score indicates a higher likelihood of occurrence. Recombination activity is shown as a percentage of wild type (\% SEM). Residues with multiple mutations are shown with both alternative variants and values. MRFmax $=0.043$ and $M R F m i n=0.004$. The full table of all protein positions can be found in Supplemental Table E1

\begin{tabular}{|c|c|c|c|}
\hline RAG1 & & & \\
\hline MRF & Residue & Assayed & Recombination activity (\%) \\
\hline 0.03 & 56 & $\mathrm{I} 56 \mathrm{~T}$ & $3.5 \pm 0.2$ \\
\hline 0.03 & 86 & K86VfsX33 & $2.7 \pm 0.3$ \\
\hline 0.014 & 99 & G99S & $113.2 \pm 3.7$ \\
\hline 0.012 & 106 & N106K & $80.4 \pm 16.4$ \\
\hline 0.043 & 108 & R108X & $1.8 \pm 0.3$ \\
\hline 0.043 & 142 & $\mathrm{R} 142 \mathrm{X}$ & $9.0 \pm 4.0$ \\
\hline 0.032 & 174 & E174SfsX27 & $0.5 \pm 0.2$ \\
\hline 0.027 & 246 & A246Tfs X17 & $0.8 \pm 0.1$ \\
\hline 0.012 & 248 & Q248X & $1.2 \pm 0.2$ \\
\hline 0.026 & 249 & H249R & $112.2 \pm 3.5$ \\
\hline 0.043 & 314 & R314W & $24.3 \pm 5.2$ \\
\hline 0.012 & 328 & C328Y & $16.0 \pm 2.9$ \\
\hline 0.03 & 383 & K383RfsX7 & $0.1 \pm 0.0$ \\
\hline 0.013 & 386 & $\mathrm{~F} 386 \mathrm{Cfs} \mathrm{X} 4<\mathrm{a} 0>$ & $0.2 \pm 0.1$ \\
\hline 0.03 & 391 & K391E & $6.5 \pm 1.6$ \\
\hline 0.043 & 394 & R394Q & $0.1 \pm 0-0.1$ \\
\hline 0.043 & 396 & R396C & $0.4-0.6 \pm 0-0.1$ \\
\hline 0.041 & 401 & S401P & $0.0 \pm 0.0$ \\
\hline 0.02 & 403 & $\mathrm{~T} 403 \mathrm{P}$ & $0.0 \pm 0.0$ \\
\hline 0.043 & 404 & R404Q & $1.2 \pm 0.1$ \\
\hline 0.043 & 410 & R410Q & $0.0 \pm 0.0$ \\
\hline 0.025 & 411 & L411P & $0.0 \pm 0.0$ \\
\hline 0.022 & 429 & D429G & $0.1 \pm 0.0$ \\
\hline 0.028 & 433 & V433 M & $0.2 \pm 0.0$ \\
\hline 0.019 & 435 & M435 V & $23.6 \pm 4.8$ \\
\hline 0.027 & 444 & A444V & $1.4 \pm 0.2$ \\
\hline 0.043 & 449 & R449K & $92.1 \pm 3.6$ \\
\hline 0.025 & 454 & L454Q & $5.4 \pm 0.7$ \\
\hline 0.019 & 458 & M458SfsX34 & $0.0 \pm 0.0$ \\
\hline 0.027 & 472 & $\mathrm{~A} 472 \mathrm{~V}$ & $0.4 \pm 0.0$ \\
\hline 0.043 & 474 & $\mathrm{R} 474 \mathrm{C}$ & $125.4 \pm 2.6$ \\
\hline 0.028 & 475 & V475AfsX17 & $0.1 \pm 0.0$ \\
\hline 0.025 & 506 & $\mathrm{~L} 506 \mathrm{~F}$ & $1.0 \pm 0.1$ \\
\hline 0.043 & 507 & R507W & $15.9 \pm 0.8$ \\
\hline 0.014 & 516 & G516A & $40.2 \pm 1.3$ \\
\hline 0.005 & 522 & W522C & $41.6 \pm 1.9$ \\
\hline 0.022 & 539 & D539V & $3.2 \pm 0.2$ \\
\hline 0.025 & 541 & L541CfsX30 & $1.2 \pm 0.9$ \\
\hline 0.043 & 559 & R559S & $1.0 \pm 0.4$ \\
\hline 0.043 & 561 & $\mathrm{R} 561 \mathrm{H}$ & $2.0 \pm 0.6$ \\
\hline 0.041 & 601 & S601P & $0.0 \pm 0.0$ \\
\hline 0.026 & 612 & H612R & $121.6 \pm 0.9$ \\
\hline 0.043 & 624 & R624H & $0.0 \pm 0.4$ \\
\hline 0.041 & 626 & S626X & $0.0 \pm 0.0$ \\
\hline 0.041 & 651 & S651P & $0.5 \pm 0.5$ \\
\hline 0.043 & 699 & R699Q,W & $45.9 \pm 1.5,19.3 \pm 1.8$ \\
\hline 0.032 & 722 & E722K & $0.0 \pm 0.2$ \\
\hline 0.012 & 730 & $\mathrm{C} 730 \mathrm{~F}$ & $0.0 \pm 0.0$ \\
\hline 0.025 & 732 & L732P & $0.0 \pm 0.0$ \\
\hline 0.043 & 737 & $\mathrm{R} 737 \mathrm{H}$ & $0.2 \pm 0.0$ \\
\hline 0.043 & 759 & $\mathrm{R} 759 \mathrm{C}$ & $17.2 \pm 3.3$ \\
\hline 0.043 & 764 & R764P & $0.0 \pm 0.0$ \\
\hline 0.008 & 768 & Y768X & $0.0 \pm 0.0$ \\
\hline 0.032 & 770 & E770K & $21.0 \pm 0.4$ \\
\hline 0.043 & 778 & R778Q,W & $8.6 \pm 1.0,4.6 \pm 0.6$ \\
\hline 0.028 & 786 & P786L & $0.0 \pm 0.1$ \\
\hline 0.03 & 820 & K820R & $117.9 \pm 6.3$ \\
\hline 0.025 & 836 & L836 V & $75.0 \pm 1.3$ \\
\hline 0.043 & 841 & R841Q,W & $0.0 \pm 0.0,10.0 \pm 0.5$ \\
\hline 0.027 & 868 & $\mathrm{~A} 868 \mathrm{~V}$ & $100.0 \pm 5.0$ \\
\hline
\end{tabular}

Table 1 (continued)

\begin{tabular}{|c|c|c|c|}
\hline 0.005 & 896 & W896R & $0.9 \pm 0.1$ \\
\hline 0.008 & 912 & Y912C & $6.9 \pm 0.4$ \\
\hline 0.005 & 959 & W959X & $0.0 \pm 0.0$ \\
\hline 0.032 & 965 & E965X & $0.0 \pm 0.0$ \\
\hline 0.043 & 973 & R973C & $0.0 \pm 0.2$ \\
\hline 0.013 & 974 & F974 L & $56.5 \pm 0.8$ \\
\hline 0.043 & 975 & R975W,Q & $57.9 \pm 1.6,53.5 \pm 3.6$ \\
\hline 0.012 & 981 & Q981P & $7.2 \pm 0.1$ \\
\hline 0.03 & 983 & K983NfsX9 & $0.1 \pm 0.0$ \\
\hline 0.03 & 992 & K992E & $9.1 \pm 1.2$ \\
\hline 0.03 & 1006 & M1006 V & $105.6 \pm 6.8$ \\
\hline \multicolumn{4}{|l|}{ RAG2 } \\
\hline 0.013 & 1 & M1T & $65.3 \pm 2.2$ \\
\hline 0.006 & 16 & Q16X & $1.7 \pm 0.4$ \\
\hline 0.038 & 35 & G35A,V & $22.1 \pm 3.1,0.4 \pm 0.3$ \\
\hline 0.023 & 39 & R39G & $0.2 \pm 0.1$ \\
\hline 0.011 & 41 & $\mathrm{C} 41 \mathrm{~W}$ & $0.2 \pm 0.4$ \\
\hline 0.017 & 62 & F62 L & $19.6 \pm 3$ \\
\hline 0.028 & 65 & D65Y & $6.8 \pm 1.2$ \\
\hline 0.023 & 73 & $\mathrm{R} 73 \mathrm{H}$ & $12.4 \pm 1.4$ \\
\hline 0.034 & 77 & T77 N & $42.6 \pm 2.7$ \\
\hline 0.038 & 95 & G95R & $0.3 \pm 0.2$ \\
\hline 0.013 & 110 & M110 L & $74.6 \pm 1.8$ \\
\hline 0.017 & 127 & K127X & $0.1 \pm 0$ \\
\hline 0.038 & 157 & G157 V & $0.4 \pm 0.2$ \\
\hline 0.03 & 160 & S160 L & $5.8 \pm 0.6$ \\
\hline 0.023 & 180 & $\mathrm{P} 180 \mathrm{H}$ & $31.1 \pm 0.5$ \\
\hline 0.019 & 195 & Y195D & $2 \pm 0.3$ \\
\hline 0.034 & 215 & $\mathrm{~T} 215 \mathrm{I}$ & $67.2 \pm 1$ \\
\hline 0.023 & 229 & R229Q,W & $8.9 \pm 1,10.5 \pm 0.5$ \\
\hline 0.023 & 253 & P253R & $95.4 \pm 2.3$ \\
\hline 0.006 & 278 & Q278X & $0.1 \pm 0.1$ \\
\hline 0.013 & 285 & M285R & $24.7 \pm 0.8$ \\
\hline 0.004 & 307 & W307X & $0.2 \pm 0.2$ \\
\hline 0.017 & 386 & F386 L & $109.1 \pm 5$ \\
\hline 0.025 & 407 & E407X & $2.9 \pm 0.4$ \\
\hline 0.004 & 416 & W416 L & $1.4 \pm 0.2$ \\
\hline 0.025 & 437 & E437K & $0.9 \pm 0.2$ \\
\hline 0.017 & 440 & K440 N & $26.7 \pm 2.4$ \\
\hline 0.013 & 443 & M443I & $0.4 \pm 0.2$ \\
\hline 0.027 & 444 & $\mathrm{I} 444 \mathrm{M}$ & $2.7 \pm 0.3$ \\
\hline 0.011 & 446 & C446W & $2.9 \pm 0.1$ \\
\hline 0.038 & 451 & G451A & $66.3 \pm 4.8$ \\
\hline 0.004 & 453 & W453R & $0.6 \pm 0.1$ \\
\hline 0.017 & 456 & A456T & $16 \pm 2.9$ \\
\hline 0.013 & 459 & M459 L & $30.8 \pm 0.6$ \\
\hline 0.034 & 474 & N474S & $97.5 \pm 5.9$ \\
\hline 0.011 & 478 & C478Y & $0.2 \pm 0.1$ \\
\hline 0.025 & 480 & E480X & $2.8 \pm 0.6$ \\
\hline 0.017 & 481 & H481P & $23.8 \pm 3.9$ \\
\hline 0.013 & 502 & M502 V & $99.6 \pm 3.4$ \\
\hline
\end{tabular}

\section{Raw Data Availability and Analysis Script}

The Supplemental "Raw_data_R_analysis_for_figures" contains all raw data and analysis methods used to produce figures (except illustrations in Figs. 1 and 6). "data_analysis.R" is an R script that contains the methods used to produce figures. Each of the input data CSV files is explained on the first usage within the analysis script. Running "data_analysis.R" from within the same directory as the associated input data CSV files will replicate analysis. 


\section{Data Visualization}

For our visualization of MRF scores, small clusters of high MRF values were more appealing than individual highly conserved residues. Therefore, we applied a $1 \%$ average filter where values were averaged over a sliding window of $\mathrm{N}$ number of residues (10 in the case of RAG1, 6 in the case of RAG2). For a clear distinction of MRF clusters, a cutoff threshold was applied at the 75th percentile (e.g., 0.0168 in RAG1) as shown in heatmaps in Figs. 1c and 6. The gene heatmaps for coding regions in RAG1 and RAG2 (Fig. 1) were populated with (i) Boolean $C$ score from population genetics data, (ii) raw MRF scores, and (iii) MRF clusters with $1 \%$ average and cutoff threshold. GraphPad Prism was used for heatmaps. The data used for heatmaps is available in Table E1 and in the supplemental R source to allow for alternative visualizations. An example of alternative output for non-R users is shown in Fig. E2. Adobe Illustrator and Photoshop were used for protein domain illustrations in Fig. 1d. Data and analysis is summarized in Fig. E1.

\section{Validation of MRF Against Functional Data}

The recombination activity of RAG1 and RAG2 was previously measured on known or candidate pathogenic variants [16-18]. Briefly, the pathogenicity of variants in RAG1 and

(a)

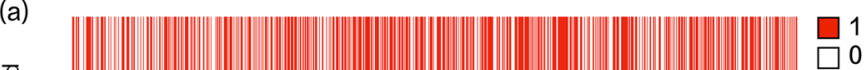

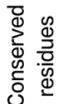

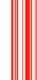

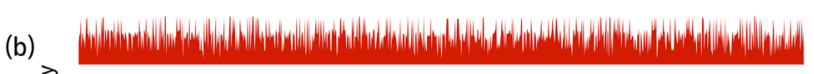

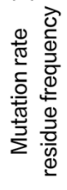

(c)

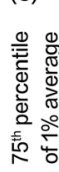

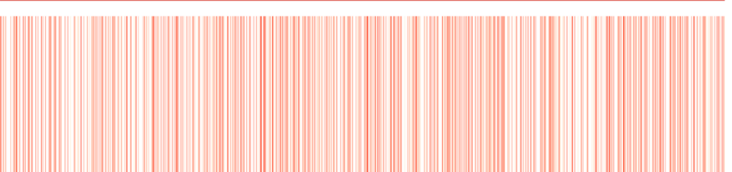

IIIII
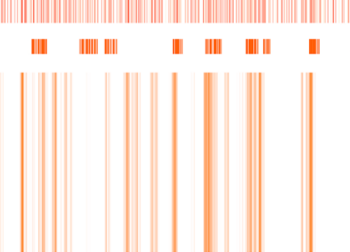

(d)

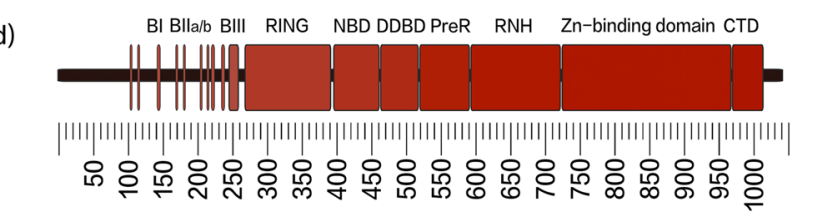

Fig. 1 RAG1 (red, left) and RAG2 (blue, right) conservation and mutation rate residue frequency. a Gene conservation score, nonconserved 0 and conserved 1 . The color indicates no known mutations in humans. b Histogram, raw MRF score; Heatmap, MRF prediction for conserved residues, graded 0 to 0.05 (scale of increasing mutation
RAG2 was measured functionally in vitro by either expression of RAG1 and RAG2 in combination with a recombination substrate plasmid containing recombination signal sequence (RSS) sites which are targeted by RAG complex during normal V(D)J recombination, or Abelson virus-transformed Rag2-/- pro-B cells with an RSS-flanked inverted GFP cassette. Recombination events were assessed by quantitative real-time PCR using comparative CT or expression of GFP evaluated by flow cytometry, respectively. The inverse score of recombination activity $(0-100 \%)$ was used to quantify pathogenicity of variants in our study. Comparison between known pathogenicity scores and MRF was done by scaling MRF scores from 0 to $100 \%$ (100\% being the highest probability of occurring as damaging).

\section{Results}

\section{RAG1 and RAG2 Conservation and Mutation Rate Residue Frequency}

Variant probability prediction is dependent on population genetics data. Our study queried GnomAD [21] to identify conserved residues using a Boolean score $C$ of 0 (present in population) or 1 (conserved). The gene-specific mutation rate $\mathrm{Mr}$ of each residue was calculated from variant allele
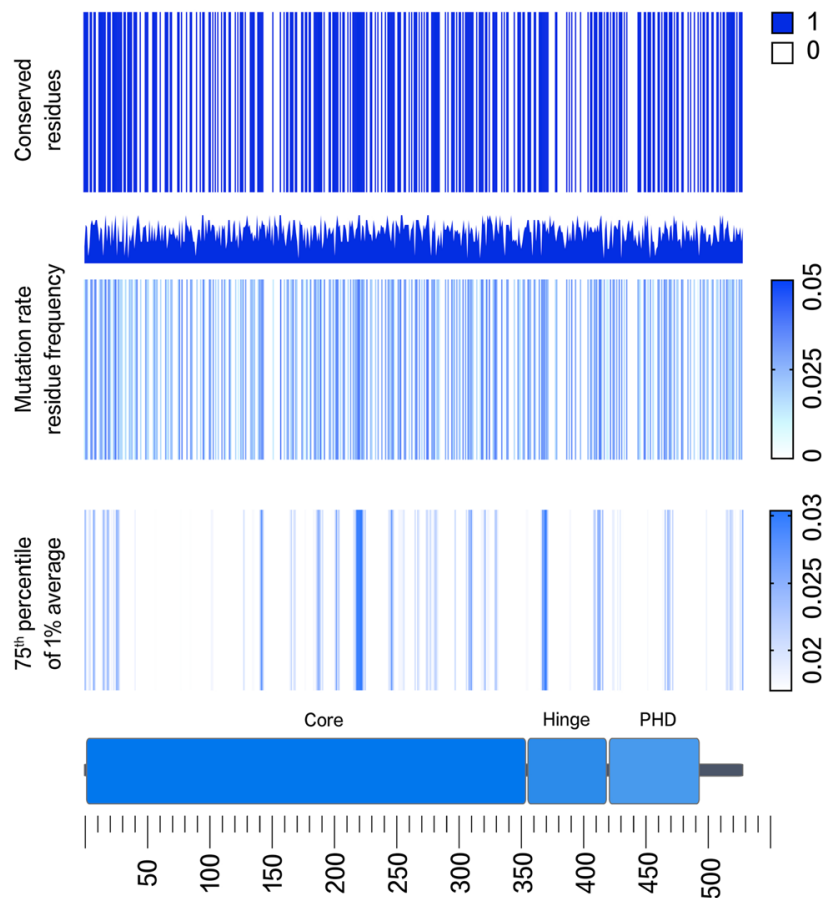

likelihood with human disease). $\mathbf{c}$ Colored bars indicate most likely clinically relevant variant clusters. MRF score averaged with $1 \%$ intervals for each gene and cutoff below the 75th percentile, graded 0 to 0.03 (noise reduction method). d Gene structure with functional domains. Full list of residues and scores available in Table E1. 
frequencies. The gene-specific residue frequency $R f$ represented the frequency of a residue occurring per gene, acquired by converting gene residue percentage (from the SMS2 tool Protein Stats) to a frequency (decimal 0-1) [24]. Together, the values were used to calculate the most probable disease-causing variants which have not yet been identified in patients. We termed the resulting score a mutation rate residue frequency, where $M R F=C \times M r \times R f$. This score represents the likelihood that a clinically relevant mutation will occur.

Figure 1 presents the most probable unidentified disease-causing variants in RAG1/2. Variants with a low MRF may still be damaging, but resources for functional validation are best spent on gene regions with high MRF. Clusters of conserved residues are shown in Fig. 1a and are generally considered important for protein structure or function. However, these clusters do not predict the likelihood of mutation. Raw MRF scores are presented in Fig. 1b. Histograms illustrate the MRF without Boolean scoring applied and Fig. 1c provides a clearer illustration of top MRF score clusters. For visualization, a noise reduction method was applied; a sliding window was used to find the average MRF per $1 \%$ interval of each gene. The resulting scores displayed in Fig. 1c contain a cutoff threshold to highlight the topscoring residues (using the 75 th percentile). Variant sites most likely to present in disease cases are identified by high MRF scoring. This model may be expanded by the addition of phenotypic or epigenetic data (Supplemental; Bayesian probability).

Table E1 provides all MRF scores for both proteins. Raw data used for calculations and the list of validated residues of RAG1 and RAG2 are available in Tables E3-E8. Table 1 shows the MRF mutation likelihood score for mutations that have also been reported as tested for recombination activity in functional assays. The likelihood of mutation does not correlate with pathogenicity; Figs. 3 and E3 show that most mutations tested had severe loss of protein function, while the likelihood of each mutation occurring in humans varied significantly. Analysis-ready files are also available in Supplemental data along with the associated $\mathrm{R}$ source file to allow for alternative visualizations as shown in Fig. E2.

\section{MRF Scores Select for Confirmed Variants in Human Disease}

We have applied MRF scores to known damaging mutations from other extensive reports in cases of human disease [12, $15,17,19,20,30-53]$ (originally compiled by Notarangelo et al. [54]). This dataset compares a total of 44 variants. We expected that functionally damaging variants (resulting in low recombination activity in vitro) that have the highest probability of occurrence would be identified with high MRF scores. MRF prediction correctly identified clinically relevant mutations in $R A G 1$ and $R A G 2$ (Fig. 2a). Variants reported on GnomAD which are clinically found to cause disease had significantly higher MRF scores than variants which have not been reported to cause disease. We observed that rare and likely mutations provided high scores while rare but unlikely or common variants had low scores (Fig. 2b).

Allele frequency is generally the single most important filtering method for rare disease in whole-genome (and exome) sequencing experiments. Variants under pressure from purifying selection are more likely to cause disease than common variants. However, most RAG mutations are rare. Therefore, allele frequencies of rare variants reported on GnomAD cannot differentially predict the likelihood of causing disease (Fig. 2b). As such, we found no significant difference between known damaging variants and those that have not yet been reported as disease causing. The comparison between Fig. 2a and $b$ illustrates the reasoning for the design of our method.

Many non-clinically reported rare variants may cause disease; the MRF score identifies the top clinically relevant candidates. Based on the frequency of protein-truncating variants in the general population, $R A G 1$ and $R A G 2$ are considered to be tolerant to the loss of one allele, as indicated by their low probability of being loss-of-function intolerant (pLI) scores of 0.00 and 0.01 , respectively [21]. This is particularly important for recessive diseases such as RAG deficiency where most new missense variants will be of unknown significance until functionally validated.

\section{Top Candidate Variants Require Validation}

Functionally, characterizing protein activity is both costly and time consuming. RAG1 and RAG2 have now been investigated by multiple functional assays for at least 110 coding variants [16-18]. In each case, researchers selected variants in $R A G 1$ and $R A G 2$ that were potentially damaging or were identified from PID patients as the most probable genetic determinant of disease. Functional assays for RAG deficiency in those cases, and generally, measured a loss of recombination activity as a percentage of wild-type function $(0-100 \%)$.

Pre-emptively, performing functional variant studies benefits those who will be identified with the same variants in the future, before the onset of disease complications. While more than 100 variants have been assayed in vitro, we calculated that only one-quarter of them are most probable candidates for clinical presentation. Figure 3 illustrates that while functional work targeted "handpicked" variants that were ultimately confirmed as damaging, many of them may be unlikely to arise based on population genetics data. Figure 3 presents, in increasing order, the number of potential variants based on the 
(a)

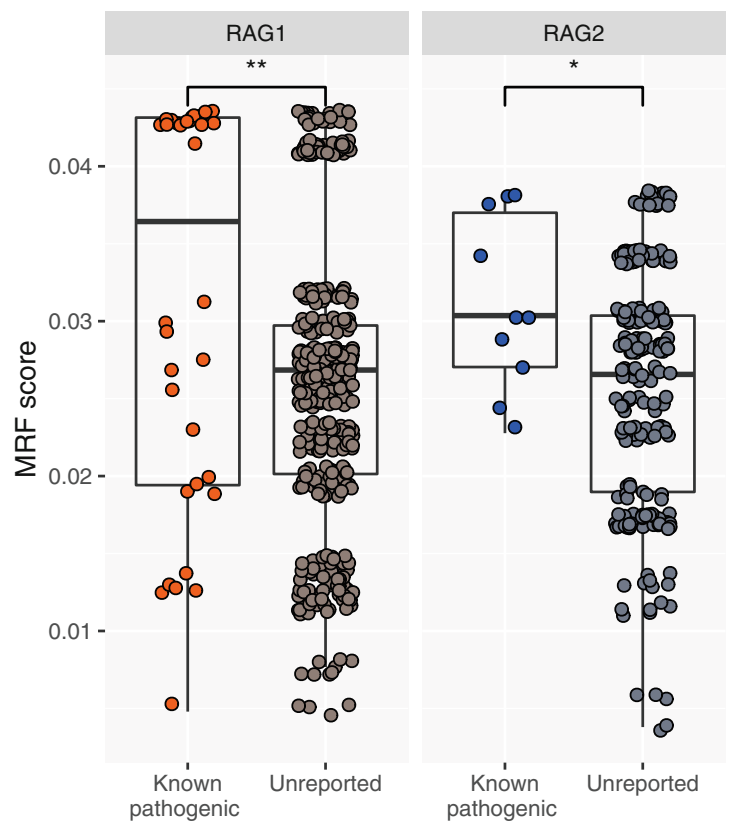

(b)

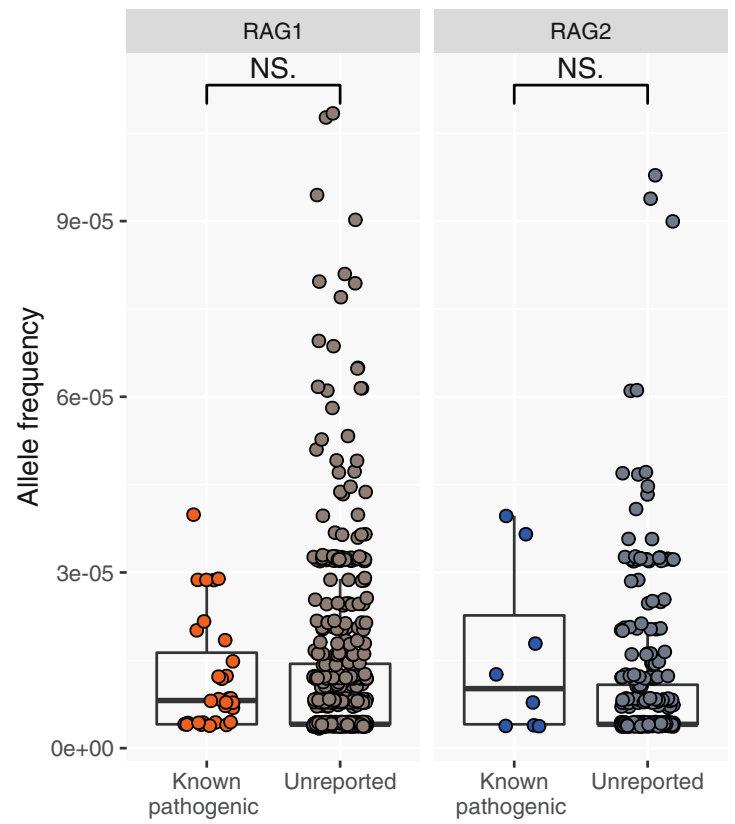

Status as a pathogenic variant and present in general population

Fig. 2 RAG1 and RAG2 MRF scores predict the likelihood of mutations that are clinically relevant. a Known damaging variants (clinically diagnosed with genetic confirmation) reported on GnomAD have significantly higher MRF scores than unreported variants. b GnomAD

likelihood of presentation and stacked by the number of variants per score category. Variants that have been measured for their loss of protein activity are colored by severity. Potential variants that remain untested are colored in grey. Only 21 of the top 66 most probable clinically relevant variants have been assayed in RAG1.

Supplemental Fig. E3 further illustrates the individual variants which have been tested functionally (the colored recombination activity subset of Fig. 3). We compared predicted MRF scores to assay measurements for 71 RAG1 and 39 RAG2 mutants. Most mutations tested showed severe loss of protein function (bottom panel of Supplemental Fig. E3), while the likelihood of each mutation occurring in humans varied significantly (top panels).

If MRF scoring was used in the same cases preemptively, the loss of investment would be minimal; only 8 variants out of 71 mutants tested had an above-average MRF score while being measured as functionally benign (a rate of $11.27 \%$ ). RAG2 had only 3 out of 39 variants (7.69\%) with an above-average MRF score while functionally benign. For the expended resources, approximately $30 \%$ more top candidates would have been tested in place of unlikely and functionally non-damaging mutations. However, the true measurement of accuracy is limited in that very few of the most likely clinically relevant variants predicted by MRF scoring have been tested to date. rare variant allele frequency $<0.0001$. No significant difference in allele frequency is found between known damaging and non-clinically reported variants. Unpaired $t$ test, RAG1 $P$ value $0.002 * *$ and RAG2 $P$ value 0.0339*. MRF, mutation rate residue frequency; ns, non-significant

\section{False Positives in Transib Domains Do Not Negatively Impact Prediction}

Adaptive immunity is considered to have evolved through jawed vertebrates after integration of the RAG transposon into an ancestral antigen receptor gene $[55,56]$. The Transib transposon is a 600 amino acid core region of RAG1 that targets RSS-like sequences in many invertebrates. A linked RAG1/ RAG2 was shown in the lower dueterostome (sea urchin), indicating an earlier common ancestor than the invertebrate [57], and more recently, a recombinatorially active RAG transposon (ProtoRAG) was found in the lower chordate amphioxus (or lancelet), the most basal extant chordate and a "living fossil of RAG" [58].

A set of conserved motifs in core RAG1 are shared with the Transib transposase, including the critical DDE residue catalytic triad (residues 603, 711, and 965) [59]. Ten RAG1 core motifs are conserved amongst a set of diverse species including human [59]. This evolutionarily conserved region is considered as most important to protein function. Therefore, we chose this region to determine if MRF scoring would have a negative impact if mutations were falsely predicted as clinically important. To assess the influence of a false-positive effect on prediction, the MRF scores for conserved residues in this group were compared with GnomAD allele frequencies. Figure 4a plots the MRF (without omitting the Boolean component $C=0$ ) for conserved Transib motif residues, non- 


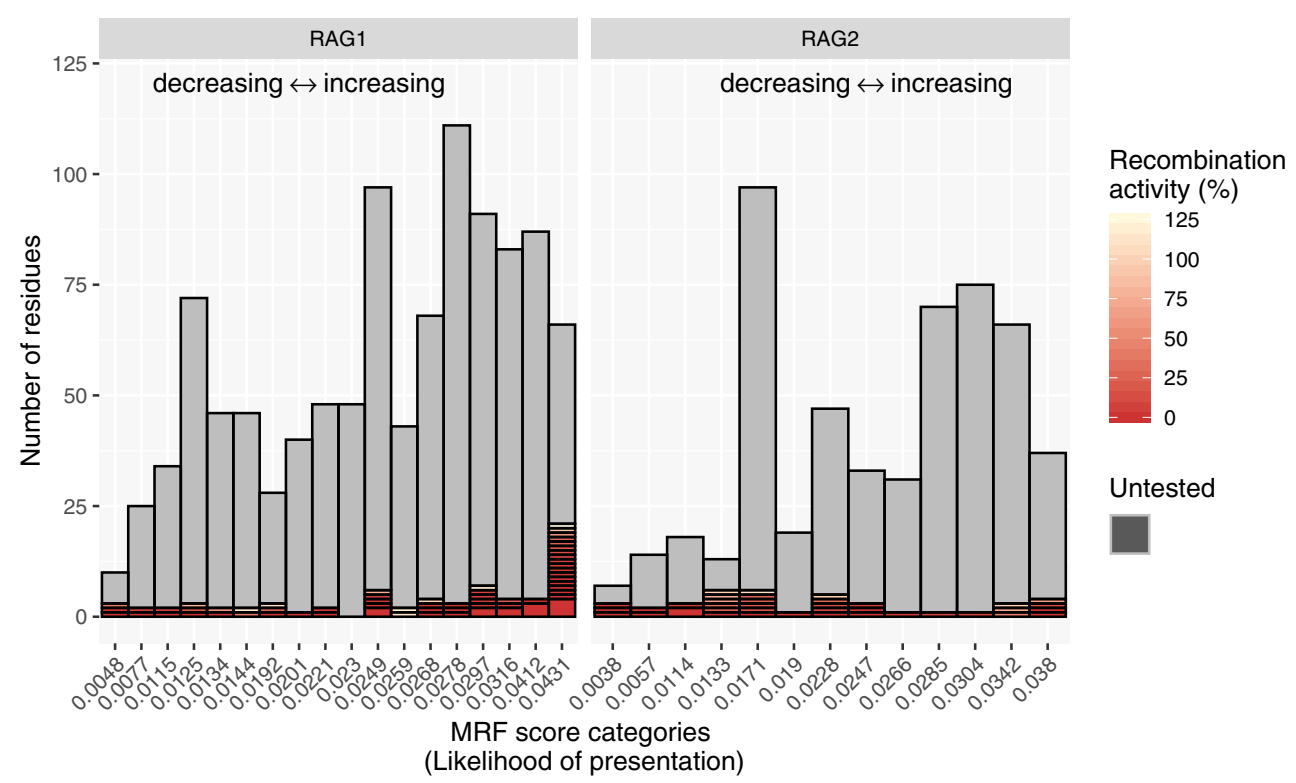

Fig. 3 RAG1 and RAG2 MRF score categories and variants assayed to date. Protein residues are ranked and stacked into categories based on their MRF score. High scores (0.043 and 0.038 in RAG1 and RAG2, respectively) represent a greater mutation likelihood. Functional assays have measured recombination activity (as its inverse; \% loss of activity) in a total of 110 mutants. The severity of protein loss of function is represented by a red gradient. Residues that have not been functionally tested are shown in grey. While many protein residues are critical to protein function, their mutation is less probable than many of the top MRF candidates. Data further expanded in Fig. E3. MRF, mutation rate residue frequency conserved Transib motif residues, and non-Transib residues. Figure $4 \mathrm{~b}$ shows the percentage of these which were reported as mutated on GnomAD. By accounting for unreported variants by applying $C>0$, the resulting effect on incorrectly scoring MRF in the conserved Transib motifs remained neutral.

\section{MRF Predicts RAG Deficiency Amongst PID Patients Harboring Rare Variants}

We have previously measured the recombination activity of RAG1 and RAG2 disease-causing variants in several patients [16]. We have compiled our own and other functional assay data from Lee et al. [17] and Tirosh et al. [18] to produce a panel of recombination activity measurements for coding variants in both RAG1 and RAG2. RAG deficiency was measured as the level of recombination potential produced by the protein complex. Each method of investigation simulated the efficiency of wild-type or mutant proteins expressed by patients for their ability to produce a diverse repertoire of $\mathrm{T}$ cell receptor (TCR) and $\mathrm{B}$ cell receptor (BCR) and coding for immunoglobulins. In functional experiments, mutant proteins were assayed for their ability to perform recombination on a substrate which mimics the RSS of TCR and BCR in comparison with wild-type protein complex (as \% SEM).

By gathering confirmed RAG deficiency cases, we compiled the MRF scores for 43 damaging RAG1 variants in 77 PID cases and 14 damaging RAG2 variants in 21 PID cases (MRF scores spanning over 22 categories). To test our method against a strong control group, we identified coding variants in patients with PID where RAG deficiency due to coding variants has been ruled out as the cause of disease. We obtained RAG1/2 variants in 558 PID patients who had their genomes sequenced as part of the NIHR BioResource - Rare Diseases study [16]. Filtering initially identified 32 variants in 166 people. This set was trimmed to contain only rare variants; 29 variants over 26 MRF scoring categories from 72 cases of non-RAG-deficient PID. The scatterplot in Fig. 5 shows that most PID cases had damaging variants with a high MRF score, while PID cases carried benign variants in RAG1/2 with lower MRF scores, i.e., an MRF $>0.04$ was seen for 31 cases of a damaging variant and only 2 cases of a non-damaging variant. Linear regression on this control group produced negative or near-zero slopes for RAG1 and RAG2, respectively. The same analysis for known damaging mutations in disease cases had significant prediction accuracy for RAG1. Analysis of RAG2 was not significant. However, the sample size to date may be too small to significantly measure RAG2 MRF scoring although a positive correlation was inferred in Fig. 5 [60]. R source and raw data can be found in Supplemental material.

\section{MRF Supplements Pathogenicity Prediction Tools for Translational Research}

CADD scoring [25] is an important bioinformatics tool that exemplifies pathogenicity prediction. While CADD is a valuable scoring method, its purpose is not to predict the 
(a)

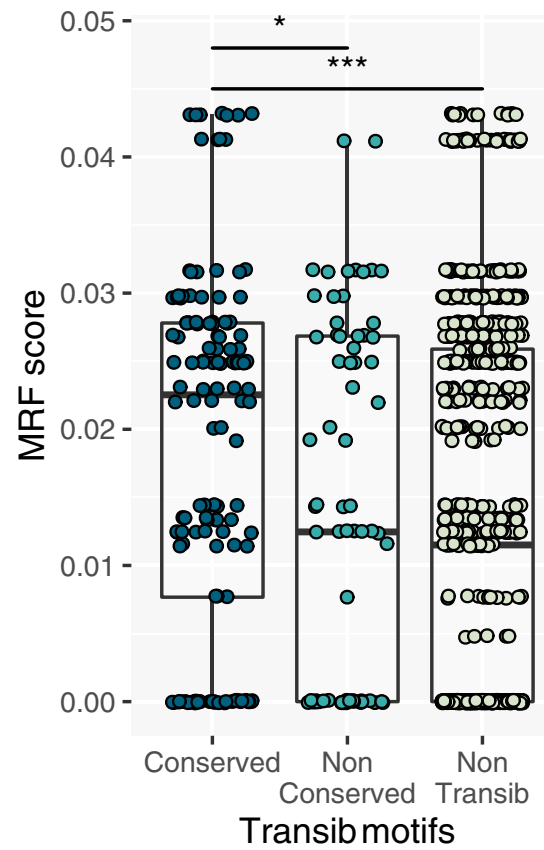

(b)

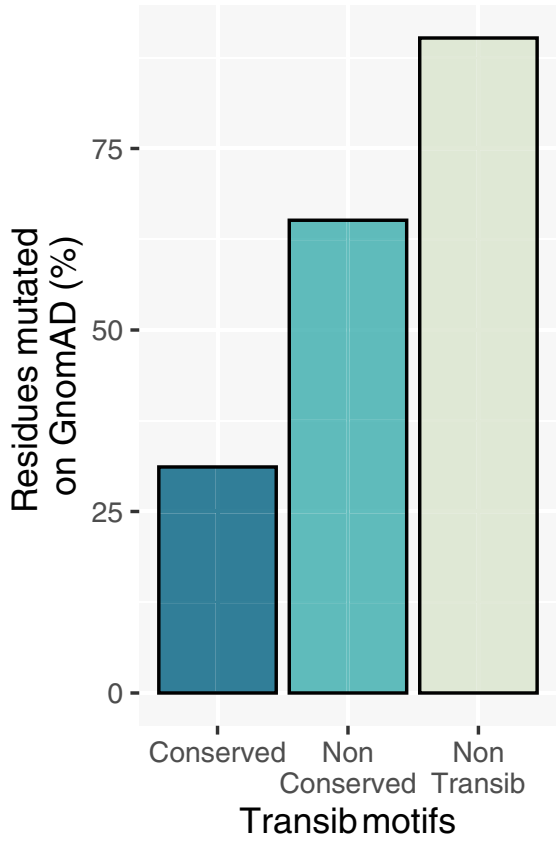

(c)

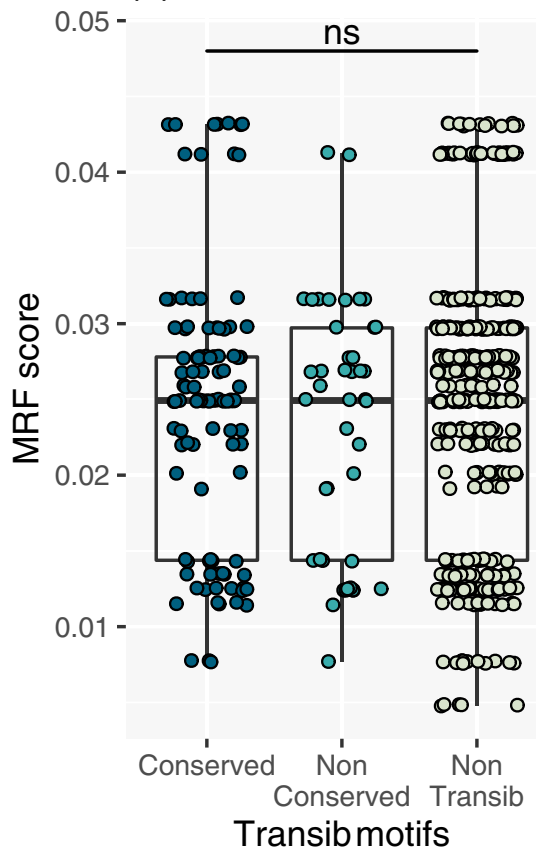

Fig. 4 False positives in Transib domains do not worsen probability prediction. The Transib domains contain critical conserved protein residues. a False positives were simulated by scoring Transib domains MRF without omitting Boolean conservation weight $C=0$. b Allele frequencies on GnomAD had conservation levels inversely proportional to simulated false-positive MRF scoring. $\mathbf{c}$ When testing for all Boolean

likelihood of variation. Similarly, MRF scoring is not a measure of pathogenicity. MRF scoring may be complemented by tools for scoring variant deleteriousness. We compare MRF to the PHRED-scaled CADD scores for all possible SNV component $C>0$ after MRF calculation, the effect of false positives remained non-significant, illustrating the non-negative impact of MRF for predicting the mutation rate. Unpaired $t$ test, $* P=0.0195$ and $* * * P<0.0001$. MRF, mutation rate residue frequency; ns, nonsignificant

positions in $R A G 1$ (Fig. 6) illustrating that pathogenicity prediction cannot account for mutation probability. Combining both methods allows researchers to identify highly probable mutations before querying predicted pathogenicity.
Fig. 5 A linear regression model of RAG1/2 MRF scoring in cases of primary immune deficiency. MRF prediction correlates with clinical presentation. Damaging variants identified in confirmed RAG deficiency cases. Nondamaging variants sourced from cases of PID with rare variants but not responsible for disease. An MRF $>0.04$ was seen for 31 cases of damaging RAG1 variants. (Slopes of RAG1: Damaging, $0.0008 *( \pm 0.0004) P<0.05$, intercept 5.82e-05***; non-damaging, $-0.0007( \pm 0.001)$. Slopes of RAG2: Damaging, 0.0023 ( \pm 0.0018 ), intercept $0.0312^{*}$; nondamaging $0.0001( \pm 0.0008)$. Source data and script in Supplemental material)

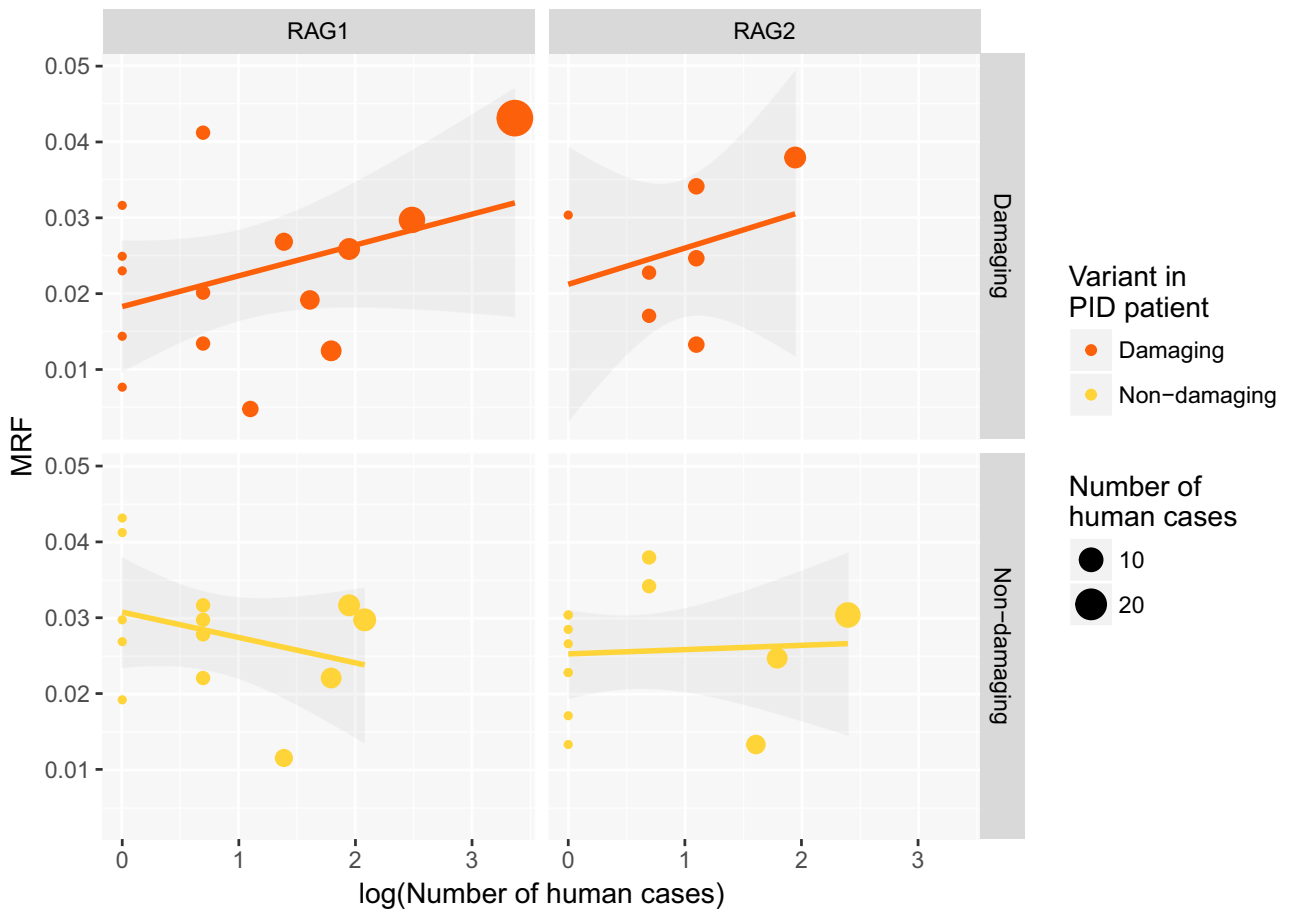


To further develop this concept, we firstly annotated variants with MRF likelihood scores and pathogenic prediction PHREDscaled CADD scores (Fig. 7) and secondly performed a manual investigation of the clinical relevance of top candidates (Table E2). We used HGMD as an unbiased source of known RAG deficiency cases in both instances. CADD score was very successful at predicting the pathogenicity of a variant, (a high-density cluster of variants with CADD scores $>25$ ) as shown in red in Fig. 7a. At about the same rate, CADD score also predicted variants as pathogenic that are, to date, unreported (as pink in Fig. 7a). Indeed, those unreported variants may very well be pathogenic. However, the likelihood of each mutation varies. As such, we developed the MRF score to account for that likelihood. As expected, the likelihood of mutations occurring that were unreported was low according to MRF (Fig. 7b, pink), while the mutations which did occur were highly enriched in at high MRF scores (Fig. 7b, red highdensity cluster $>0.043$ ). Combining mutation prediction (MRF) with pathogenicity prediction (tools like CADD) increases the accuracy of pre-emptively targeting clinically relevant variants. Figure $7 \mathrm{c}$ shows that while the number of variants presented to date is relatively small, they already account for $36 \%$ of the top MRF score candidates.

\section{Discussion}

Determining disease-causing variants for functional analysis typically aims to target conserved gene regions. On GnomAD,
$56 \%$ of RAG1 (approx. 246,000 alleles) is conserved with no reported variants. Functional validation of unknown variants in genes with this level purifying selection is generally infeasible. Furthermore, we saw that a vast number of candidates are "predicted pathogenic" by commonly used pathogenicity tools, which may indeed be damaging but unlikely to occur. To overcome the challenge of manual selection, we quantified the likelihood of mutation for each candidate variant.

Targeting clearly defined regions with high MRF scores allows for functional validation studies tailored to the most clinically relevant protein regions. An example of high MRF score clustering occurred in the RAG1 catalytic RNase H (RNH) domain at p.Ser638-Leu658 which is also considered a conserved Transib motif.

While many hypothetical variants with low MRF scores may be uncovered as functionally damaging, our findings suggest that human genomic studies will benefit by first targeting variants with the highest probability of occurrence (gene regions with high MRF). Table E1 lists the values for calculated MRFs for RAG1 and RAG2.

We have presented a basic application of MRF scoring for RAG deficiency. The method can be applied to genome wide. This can include phenotypically derived weights to targetcandidate genes or tissue-specific epigenetic features. In the state presented here, MRF scores are used for pre-clinical studies. A more advanced development may allow for use in single cases. During clinical investigations using personalized analysis of patient data, further scoring methods may be applied based on disease features. A patient
Fig. 6 RAG1 PHRED-scaled CADD score versus GnomAD conservation rate and MRF score. Allele frequency conservation rate (top) is vastly important for identifying critical structural and functional protein regions. The impact of mutation in one of these conserved regions is often estimated using CADD scoring (middle). CADD score heatmap is aligned by codon and separated into three layers for individual nucleotide positions. The MRF score (bottom) (visualized using the 75 th percentile with $1 \%$ averaging) highlights protein regions that are most likely to present clinically and may require preemptive functional investigation

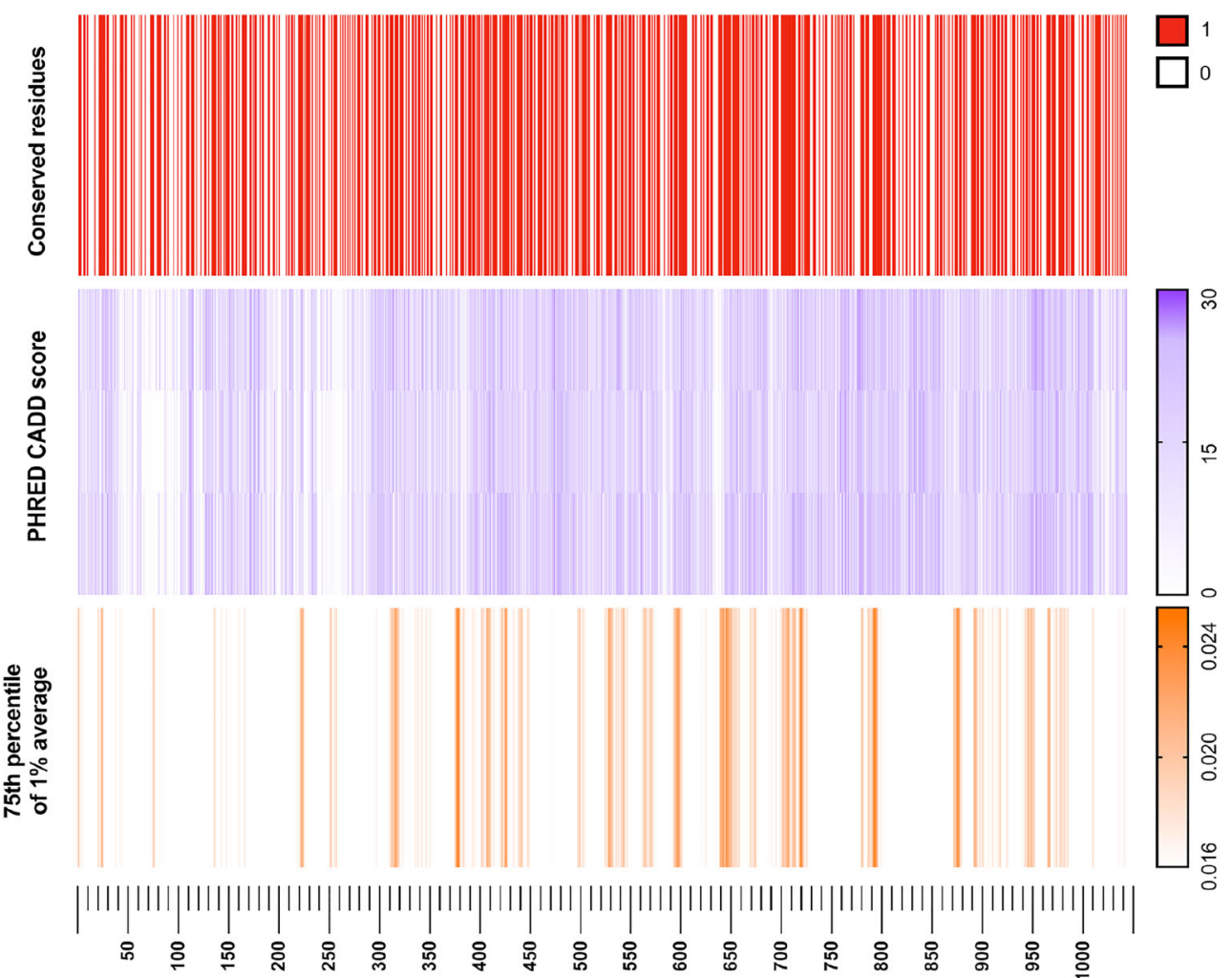


Fig. 7 RAG1 PHRED-scaled CADD score versus MRF score against HGMD data. a A high CADD score is a predictor of deleteriousness. Both reported (red) and non-reported residues (pink) have a high density of high CADD score. b MRF scores only show a high-density cluster for high-likelihood variants, reflected by the high MRF score observed for known RAG deficiency variants. The number of pathogenic variants is outweighed by conserved residues; a, b shows the density of scores to normalize between groups. AUC overlap difference in CADD score of $21.43 \%$ and MRF score of $74.28 \%$ (above intersects $>22.84$ and $>0.0409$, in $\mathbf{a}$ and $\mathbf{b}$ respectively). $\mathbf{c}$ The number of residues per MRF category shows that disease reported on HGMD accounts for $36 \%$ of top MRF candidates. AUC, area under curve; CADD, Combined Annotation Dependent Depletion; HGMD, Human Gene Mutation Database (a)

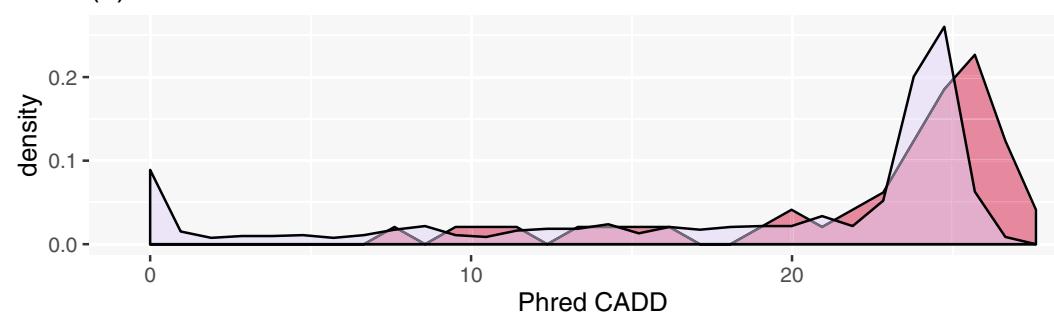

(b)

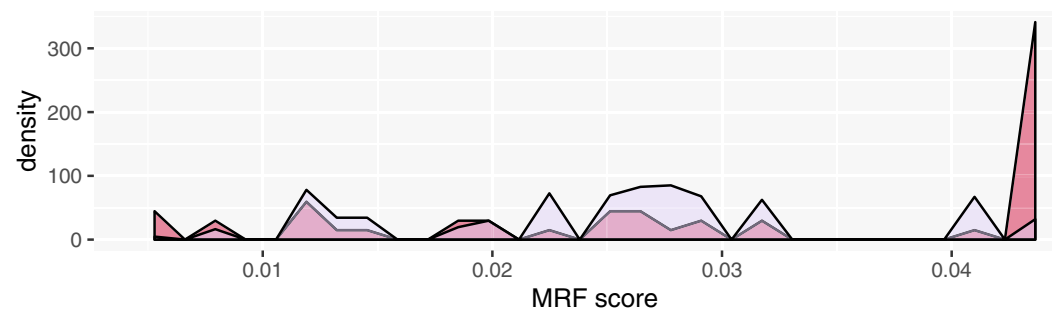

(c)

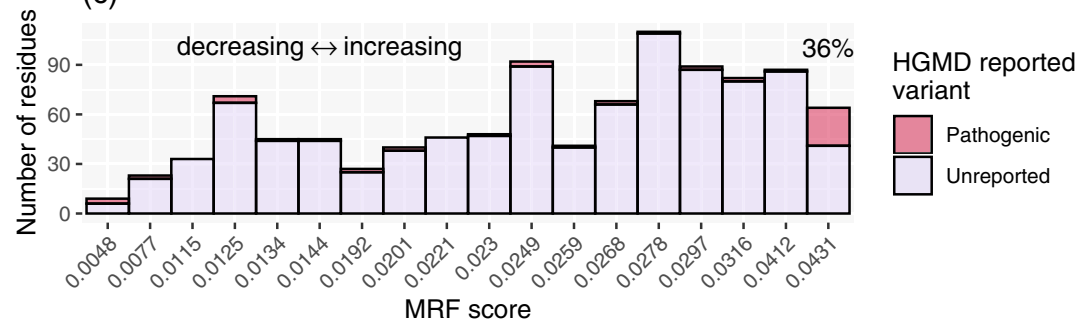

phenotype can contribute a weight based on known genotype correlations separating primary immunodeficiencies or autoinflammatory diseases [6]. For example, a patient with autoinflammatory features may require a selection that favors genes associated with proinflammatory diseases such as $M E F$ Vand TNFAIP3, whereas a patient with mainly immunodeficiency may have preferential scoring forgenes such as $B T K$ and $D O C K 8$. In this way, a check-list of most likely candidates can be confirmed or excluded by whole genome or panel sequencing. However, validation of these expanded implementations requires a deeper consolidation of functional studies than is currently available.

Havrilla et al. [61] have recently developed a method with similar possible applications for human health mapping constrained coding regions. Their study employed a method that included weighting by sequencing depth. Similarly, genomewide scoring may benefit from mutation significance cutoff, which is applied for tools such as CADD, PolyPhen-2, and SIFT [62]. We have not included an adjustment method as our analysis was gene-specific but implementation is advised when calculating genome-wide MRF scores.

The MRF score was developed to identify the topmost probable variants thathave the potential to cause disease. Itisnotapredictorof pathogenicity. However, MRF may contribute to disease prediction; a clinician may ask for the likelihood of RAG deficiency (or any other Mendelian disease of interest) prior to examination (Supplemental)[68].
Predicting the likelihood of discovering novel mutations has implications in genome-wide association studies (GWAS). Variants with low minor allele frequencies have a low discovery rate and low probability of disease association [63], an important consideration for rare diseases such as RAG deficiency. An analysis of the NHGRI-EBI catalogue data highlighted diseases whose average risk allele frequency was low [63]. Autoimmune diseases had risk allele frequencies considered low at approximately 0.4. Without a method to rank most probable novel disease-causing variants, it is unlikely that GWAS will identify very rare disease alleles (with frequencies $<0.001$ ). It is conceivable that a number of rare immune diseases are attributable to polygenic rare variants. However, evidence for lowfrequency polygenic compounding mutations will not be available until large, accessible genetics databases are available, exemplified by the NIHR BioResource Rare Diseases study [16]. An Interesting consideration when predicting probabilities of variant frequency is that of protective mutations. Disease risk variants are quelled at low frequency by negative selection, while protective variants may drift at higher allele frequencies [64].

The cost-effectiveness of genomic diagnostic tests is already outperforming traditional, targeted sequencing [1]. Even with substantial increases in data sharing capabilities and adoption of clinical genomics, rare diseases due to variants of unknown significance and low allele frequencies will remain non-actionable until reliable predictive genomics 
practices are developed. Bioinformatics as a whole has made staggering advances in the field of genetics [65]. Challenges that remain unsolved, hindering the benefit of national or global genomics databases, include DNA data storage and random access retrieval [66], data privacy management [67], and predictive genomics analysis methods. Variant filtration in rare disease is based on reference allele frequency, yet the result is not clinically actionable in many cases. Development of predictive genomics tools may provide a critical role for single-patient studies and timely diagnosis [23].

\section{Conclusion}

We provide a list of amino acid residues for RAG1 and RAG2 that have not been reported to date but are most likely to present clinically as RAG deficiency. This method may be applied to other diseases with hopes of improving preparedness for clinical diagnosis.

Acknowledgments We gratefully acknowledge the participation of all NIHR BioResource volunteers and thank the NIHR BioResource centers and staff for their contribution. We thank the National Institute for Health Research and NHS Blood and Transplant.

Authorship Contributions Dylan Lawless analyzed data and conceived and wrote the manuscript; Hana Allen Lango analyzed NIHR genomic data; James Thaventhiran provided genomic data; NIHR BioResourceRare Diseases Consortium provided genomic data; Rashida Anwar wrote the manuscript; Jolan E. Walter provided clinical genomics data and wrote the manuscript; Jacques Fellay wrote the manuscript; Sinisa Savic conceived and wrote the manuscript.

Funding This work is funded by the University of Leeds 110 Anniversary Research Scholarship and by the National Institute for Health Research (NIHR, grant number RG65966). This work was also supported by the National Institutes of Health (sub-R01AI100887-05 to J.E.W.) and Robert A. Good Endowment, the University of South Florida (to J.E.W.).

\section{Compliance with Ethical Standards}

Ethics Statement The study was performed in accordance with the Declaration of Helsinki. The NIHR BioResource projects were approved by Research Ethics Committees in the UK and appropriate national ethics authorities in non-UK enrolment centers.

Conflict of Interest The authors declare that they have no conflict of interest.

Disclaimer The views expressed are those of the author(s) and not necessarily those of the NHS, the NIHR, or the Department of Health.

Open Access This article is distributed under the terms of the Creative Commons Attribution 4.0 International License (http:// creativecommons.org/licenses/by/4.0/), which permits unrestricted use, distribution, and reproduction in any medium, provided you give appropriate credit to the original author(s) and the source, provide a link to the Creative Commons license, and indicate if changes were made.

\section{References}

1. Payne K, Gavan SP, Wright SJ, Thompson AJ. Cost-effectiveness analyses of genetic and genomic diagnostic tests. Nat Rev Genet. 2018;19(4):235-46.

2. Kwan A, Abraham RS, Currier R, Brower A, Andruszewski K, Abbott JK, et al. Newborn screening for severe combined immunodeficiency in 11 screening programs in the united states. Jama. 2014;312(7):729-38.

3. Alexander Liggett L, Sharma A, De S, DeGregori J. Conserved patterns of somatic mutations in human peripheral blood cells. bioRxiv. 2017. https://doi.org/10.1101/208066.

4. Clark SJ, Argelaguet R, Kapourani C-A, Stubbs TM, Lee HJ, AldaCatalinas $\mathrm{C}$, et al. scnmt-seq enables joint profiling of chromatin accessibility dna methylation and transcription in single cells. Nat Commun. 2018;9(1):781

5. Bartha I, di Iulio J, Venter JC, Telenti A. Human gene essentiality. Nat Rev Genet pages nrg-2017. 2017;19(1):51-62.

6. Picard C, Gaspar HB, Al-Herz W, Bousfiha A, Casanova J-L, Chatila $\mathrm{T}$, et al. International union of immunological societies: 2017 primary immunodeficiency diseases committee report on inborn errors of immunity. J Clin Immunol. 2018;38(1):96-128.

7. Conley ME, Casanova J-L. Discovery of single-gene inborn errors of immunity by next generation sequencing. Curr Opin Immunol. 2014;30:17-23.

8. Schatz DG, Oettinger MA, Baltimore D. The $v(d) j$ recombination activating gene, rag-1. Cell. 1989;59(6):1035-48.

9. Oettinger MA, Schatz DG, Gorka C, Baltimore D. Rag-1 and rag-2, adjacent genes that synergistically activate $\mathrm{v}(\mathrm{d}) \mathrm{j}$ recombination. Science. 1990;248(4962):1517-23.

10. Mombaerts P, Iacomini J. Randall S Johnson, Karl Herrup, Susumu Tonegawa, and Virginia E Papaioannou. Rag-1-deficient mice have no mature b and t lymphocytes. Cell. 1992;68(5):869-77.

11. Shinkai Y, Lam K-P, Oltz EM, Stewart V, Mendelsohn M, Charron $\mathrm{J}$, et al. Rag-2-deficient mice lack mature lymphocytes owing to inability to initiate $\mathrm{v}$ (d) j rearrangement. Cell. 1992;68(5):855-67.

12. Schwarz K, Gauss GH, Ludwig L, Pannicke U, Li Z, Lindner D, et al. Rag mutations in human $b$ cell-negative scid. Science. 1996;274(5284):97-9.

13. de Saint-Basile G, Le Deist F, De Villartay JP, Cerf-Bensussan N, Journet $\mathrm{O}$, Brousse N, et al. Restricted heterogeneity of t lymphocytes in combined immunodeficiency with hypereosinophilia (omenn's syndrome). J Clin Invest. 1991;87(4):1352-9.

14. Rieux-Laucat F, Bahadoran P, Brousse N, Selz F, Fischer A, Le Deist $F$, et al. Highly restricted human $t$ cell repertoire in peripheral blood and tissue-infiltrating lymphocytes in omenn's syndrome. J Clin Invest. 1998;102(2):312-21.

15. Villa A, Santagata S, Bozzi F, Giliani S, Frattini A, Imberti L, et al. Partial v (d) j recombination activity leads to omenn syndrome. Cell. 1998;93(5):885-96.

16. Lawless D, Geier CB, Farmer JR, Allen HL, Thwaites D, Atschekzei F, et al. Prevalence and clinical challenges among adults with primary immunodeficiency and recombinationactivating gene deficiency. J Allergy Clin Immunol. 2018;141(6): 2303-6.

17. Lee YN, Frugoni F, Dobbs K, Walter JE, Giliani S, Gennery AR, et al. A systematic analysis of recombination activity and genotypephenotype correlation in human recombination-activating gene 1 deficiency. J Allergy Clin Immunol. 2014;133(4):1099-108.

18. Tirosh I, Yamazaki Y, Frugoni F, Ververs FA, Allenspach EJ, Zhang $\mathrm{Y}$, et al. Recombination activity of human rag2 mutations and correlation with the clinical phenotype. J Allergy Clin Immunol. 2018;143(2):726-35.

19. Walter JE, Rosen LB, Csomos K, Rosenberg JM, Mathew D, Keszei M, et al. Broad-spectrum antibodies against self-antigens 
and cytokines in rag deficiency. J Clin Invest. 2015;125(11):413548.

20. Schuetz C, Huck K, Gudowius S, Megahed M, Feyen O, Hubner B. Dominik T Schneider, Burkhard Manfras, Ulrich Pannicke, Rein Willemze, et al. An immunodeficiency disease with rag mutations and granulomas. N Engl J Med. 2008;358(19):2030-8.

21. Lek M, Karczewski KJ, Minikel EV, Samocha KE, Banks E, Fennell T, et al. Analysis of protein-coding genetic variation in 60, 706 humans. Nature. 2016;536(7616):285

22. John T, Walter JE, Schuetz C, Chen K, Abraham RS, Bonfim C, et al. Unrelated hematopoietic cell transplantation in a patient with combined immunodeficiency with granulomatous disease and autoimmunity secondary to rag deficiency. J Clin Immunol. 2016;36(7):725-32.

23. Casanova J-L, Conley ME, Seligman SJ, Abel L, Notarangelo LD. Guidelines for genetic studies in single patients: lessons from primary immunodeficiencies. J Exp Med, pages jem-20140520. 2014;211(11):2137.

24. Paul Stothard. The sequence manipulation suite: Javascript programs for analyzing and formatting protein and dna sequences. University of Alberta, Education and Research Archive, 2000.

25. Kircher M, Witten DM, Jain P, O'roak BJ, Cooper GM, Shendure J. A general framework for estimating the relative pathogenicity of human genetic variants. Nat Genet. 2014;46(3):310.

26. Peter D, Stenson MM, Ball EV, Shaw K, Phillips AD, Cooper DN. The human gene mutation database: building a comprehensive mutation repository for clinical and molecular genetics, diagnostic testing and personalized genomic medicine. Hum Genet. 2014;133(1): $1-9$.

27. Heng R, Melissa G. Chambers, Tian-Min Fu, Alexander B Tong, Maofu Liao, and Hao Wu. Molecular mechanism of v (d) j recombination from synaptic rag1-rag2 complex structures. Cell. 2015;163(5):1138-52.

28. Humphrey W, Dalke A, Schulten K. VMD - Visual Molecular Dynamics. J Mol Graph. 1996;14:33-8.

29. John Stone. An efficient library for parallel ray tracing and animation. Master's thesis, Computer Science Department, University of Missouri-Rolla, 1998.

30. Villa A, Sobacchi C. Luigi D Notarangelo, Fabio Bozzi, Mario Abinun, Tore G Abrahamsen, Peter D Arkwright, Michal Baniyash, Edward G Brooks, Mary Ellen Conley, et al. V (d) j recombination defects in lymphocytes due torag mutations: severe immunodeficiency with a spectrum of clinical presentations. Blood. 2001;97(1):81-8.

31. Abolhassani H, Wang N, Aghamohammadi A, Rezaei N. Yu Nee Lee, Francesco Frugoni, Luigi D Notarangelo, Qiang PanHammarström, and Lennart Hammarström. A hypomorphic recombination-activating gene $1(\operatorname{rag} 1)$ mutation resulting in a phenotype resembling common variable immunodeficiency. J Allergy Clin Immunol. 2014;134(6):1375-80.

32. Kutukculer N, Gulez N, Karaca NE, Aksu G, Berdeli A. Novel mutations and diverse clinical phenotypes in recombinase-activating gene 1 deficiency. Ital J Pediatr. 2012;38(1):8.

33. Sobacchi C, Marrella V, Rucci F, Vezzoni P, Villa A. Rag- dependent primary immunodeficiencies. Hum Mutat. 2006;27(12):117484.

34. Jeroen G, Noordzij JG, de Bruin-Versteeg S, Verkaik NS, Vossen $\mathrm{JM}$, de Groot R, et al. The immunophenotypic and immunogenotypic b-cell differentiation arrest in bone marrow of rag-deficient scid patients corresponds to residual recombination activities of mutated rag proteins. Blood. 2002;100(6):2145-52.

35. Crestani E, Choo S, Frugoni F. Yu Nee Lee, Stephanie Richards, Joanne Smart, and Luigi D Notarangelo. Rag1 reversion mosaicism in a patient with omenn syndrome. J Clin Immunol. 2014;34(5): $551-4$.
36. Dalal I, Tabori U, Bielorai B, Golan H, Rosenthal E, Amariglio N, et al. Evolution of a tb-scid into an omenn syndrome phenotype following parainfluenza 3 virus infection. Clin Immunol. 2005;115(1):70-3.

37. Kuijpers TW, Jspeert HI, van Leeuwen EMM, Jansen MH, Hazenberg MD, Weijer KC, et al. Idiopathic cd4+ t lymphopenia without autoimmunity or granulomatous disease in the slipstream of rag mutations. Blood, pages blood-2011. 2011;117(22):5892-6.

38. Gruber TA, Shah AJ, Hernandez M, Crooks GM, Abdel-Azim H, Gupta S, et al. Clinical and genetic heterogeneity in omenn syndrome and severe combined immune deficiency. Pediatr Transplant. 2009;13(2):244-50.

39. De Ravin SS, Cowen EW, Zarember KA, Whiting-Theobald NL, Kuhns DB, Sandler NG, et al. Hypomorphic rag mutations can cause destructive midline granulomatous disease. Blood. 2010;116(8):1263-71.

40. Buchbinder D, Baker R, Lee YN, Ravell J, Zhang Y, McElwee J, et al. Identification of patients with rag mutations previously diagnosed with common variable immunodeficiency disorders. J Clin Immunol. 2015;35(2):119-24.

41. Felgentreff K, Perez-Becker R, Speckmann C, Schwarz K, Kalwak $\mathrm{K}$, Markelj G, et al. Clinical and immunological manifestations of patients with atypical severe combined immunodeficiency. Clin Immunol. 2011;141(1):73-82.

42. Reiff A, Bassuk AG, Church JA, Campbell E, Bing X, Ferguson PJ. Exome sequencing reveals rag1 mutations in a child with autoimmunity and sterile chronic multifocal osteomyelitis evolving into disseminated granulomatous disease. J Clin Immunol. 2013;33(8): 1289-92.

43. Corneo B, Moshous D, Güngör T, Wulffraat N, Philippet P, Le Deist $\mathrm{F}$, et al. Identical mutations in rag1 or rag2 genes leading to defective $v$ (d) $j$ recombinase activity can cause either tb-severe combined immune deficiency or omenn syndrome. Blood. 2001;97(9):2772-6.

44. Asai E, Wada T, Sakakibara Y, Toga A, Toma T, Shimizu T, et al. Analysis of mutations and recombination activity in rag-deficient patients. Clin Immunol. 2011;138(2):172-7.

45. Kato T, Crestani E, Kamae C, Honma K, Yokosuka T, Ikegawa T, et al. Rag1 deficiency may present clinically as selective iga deficiency. J Clin Immunol. 2015;35(3):280-8.

46. Yu X, Jorge R, Almeida SD, van der Burg M, De Ravin SS, Malech $\mathrm{H}$, et al. Human syndromes of immunodeficiency and dysregulation are characterized by distinct defects in t-cell receptor repertoire development. J Allergy Clin Immunol. 2014;133(4):1109-15.

47. De Villartay J-P, Lim A, Al-Mousa H, Dupont S, DéchanetMerville J, Coumau-Gatbois E, et al. A novel immunodeficiency associated with hypomorphic rag 1 mutations and $\mathrm{cmv}$ infection. $\mathrm{J}$ Clin Invest. 2005;115(11):3291-9.

48. Zhang J, Quintal L, Atkinson A, Williams B, Grunebaum E, Roifman CM. Novel rag1 mutation in a case of severe combined immunodeficiency. Pediatrics. 2005;116(3):e445-9.

49. Henderson LA, Frugoni F, Hopkins G, Boer H, Sung-YunPai YNL, Walter JE, et al. Expanding the spectrum of recombinationactivating gene 1 deficiency: a family with early-onset autoimmunity. J Allergy Clin Immunol. 2013;132(4):969-71.

50. Avila EM, Uzel G, Hsu A, Milner JD, Turner ML, Pittaluga S, et al. Highly variable clinical phenotypes of hypomorphic rag1 mutations. Pediatrics. 2010;126(5):e1248-52.

51. Riccetto AGL, Buzolin M, Fernandes JF, Raina FT, Castro MLR Bd, Silva MTN, et al. Compound heterozygous rag2 mutations mimicking hyper igm syndrome. J Clin Immunol. 2014;34(1):7-9.

52. CarlosAGomez LMP, Villa A, Bozzi F, Sobacchi C, GBrooks E, Notarangelo LD, et al. Mutations in conserved regions of the predicted rag2 kelch repeats block initiation of $\mathrm{v}(\mathrm{d}) \mathrm{j}$ recombination and result in primary immunodeficiencies. Mol Cell Biol. 2000;20(15):5653-64. 
53. Chou J, Hanna-Wakim R, Tirosh I, Kane J, Fraulino D, Lee YN, et al. A novel homozygous mutation in recombination activating gene 2 in 2 relatives with different clinical phenotypes: Omenn syndrome and hyper-igm syndrome. J Allergy Clin Immunol. 2012;130(6):1414-6.

54. Luigi D, Notarangelo M-SK, Walter JE, Lee YN. Human rag mutations: biochemistry and clinical implications. Nat Rev Immunol. 2016;16(4):234.

55. Agrawal A, Eastman QM, Schatz DG. Transposition mediated by rag1 and rag2 and its implications for the evolution of the immune system. Nature. 1998;394(6695):744.

56. Hiom K, Melek M, Gellert M. Dna transposition by the rag1 and rag2 proteins: a possible source of oncogenic translocations. Cell. 1998;94(4):463-70.

57. Fugmann SD, Messier C, Novack LA, Cameron RA, Rast JP. An ancient evolutionary origin of the $\operatorname{rag} 1 / 2$ gene locus. Proc Natl Acad Sci U S A. 2006;103(10):3728-33.

58. Huang S, Tao X, Yuan S, Zhang Y, Li P, ABeilinson H, et al. Discovery of an active rag transposon illuminates the origins of $\mathrm{V}$ (d) j recombination. Cell. 2016;166(1):102-14.

59. Kapitonov VV, Jurka J. Rag 1 core and v (d) j recombination signal sequences were derived from transib transposons. PLoS Biol. 2005;3(6):e181.

60. Altman DG, Bland JM. Statistics notes: absence of evidence is not evidence of absence. Bmj. 1995;311(7003):485.

61. Havrilla JM, Pedersen BS, Layer RM, Quinlan AR. A map of constrained coding regions in the human genome. bioRxiv. 2017. https://doi.org/10.1101/220814.
62. Itan Y, Shang L, Boisson B, Ciancanelli MJ, Markle JG, MartinezBarricarte R, et al. The mutation significance cutoff: gene-level thresholds for variant predictions. Nat Methods. 2016;13(2):109.

63. Kido T, Sikora-Wohlfeld W, Kawashima M, Kikuchi S, Kamatani N, Patwardhan A, et al. Are minor alleles more likely to be risk alleles? BMC Med Genet. 2018;11(1):3.

64. Chan Y, Lim ET, Sandholm N, Wang SR, McKnight AJ, Ripke S, et al. An excess of risk-increasing low-frequency variants can be a signal of polygenic inheritance in complex diseases. Am J Hum Genet. 2014;94(3):437-52.

65. Libbrecht MW, Noble WS. Machine learning applications in genetics and genomics. Nat Rev Genet. 2015;16(6):321.

66. Lee O, Ang SD, Chen Y-J, Lopez R, Yekhanin S, Makarychev K, et al. Scaling up dna data storage and random access retrieval. bioRxiv. 2017. https://doi.org/10.1101/114553.

67. Huang Z, Ayday E, Lin H, Aiyar RS, Molyneaux A, Xu Z, et al. A privacy-preserving solution for compressed storage and selective retrieval of genomic data. Genome Res. 2016;26(12):10):1687-96.

68. Kumánovics A, Lee YN, Close DW, Coonrod EM, Ujhazi B, Chen $\mathrm{K}$, et al. Estimated disease incidence of $\operatorname{rag} 1 / 2$ mutations: a case report and querying the exome aggregation consortium. J Allergy Clin Immunol. 2017;139(2):690-2.

Publisher's Note Springer Nature remains neutral with regard to jurisdictional claims in published maps and institutional affiliations. 